\title{
Adhesión de láminas de caucho de llantas de desecho con modificación de superficie usando poliuretano como adhesivo y silanos como promotores de adhesión
}

\author{
Oscar Y. Buitrago-Suescún' \\ (1) Programa de Ingeniería Industrial. Universidad Militar Nueva Granada. Bogotá Colombia. \\ correo-e: oscar.buitrago@unimilitar.edu.co
}

Recibido Abr. 28, 2021; Aceptado Jun. 29, 2021; Versión final Ago. 13, 2021, Publicado Dic. 2021

\begin{abstract}
Resumen
En el presente estudio se examina el procedimiento de adhesión de láminas elaboradas con caucho de llantas desechadas usando el adhesivo poliuretano (PU). Adicionalmente, se evalúa la aplicación de una capa de elastómero sobre la superficie. Se elaboraron probetas para realizar tratamientos superficiales con ácido tricloroisocianúrico y permanganato de potasio. Se usaron tres promotores de adhesión: 1) diisocianato de tolueno, 2) 3-Aminopropiltrietoxisilano y 3) viniltrimetoxisilano. Las probetas se adhirieron y fueron sometidas a ensayos de tracción al pelado para determinar cuál es el mejor procedimiento de unión. Se realizaron pruebas de adhesión y análisis de caracterización con espectrometría infrarroja con transformada de Fourier, microscopía electrónica de barrido y energía dispersiva de rayos X. El mejor resultado se obtiene tratando la superficie con ácido tricloroisocianúrico. Se concluye que este tratamiento resulta efectivo para incrementar los valores de adherencia, tanto entre láminas unidas mediante PU como entre láminas y recubrimiento elastómerico.
\end{abstract}

\section{Adhesion of surface-modified waste tire rubber sheets by using polyurethane adhesive and silane adhesion promoters}

\begin{abstract}
The present study aimed to examine the procedure for adhesion of sheets extracted from waste tire rubber by using polyurethane (PU) as an adhesive and by applying an elastomer layer to the surface. Surface treatments were performed with trichloroisocyanuric acid and potassium permanganate. Three adhesion promoters were used: 1) toluene diisocyanate, 2) 3-aminopropyltriethoxysilane, and 3) vinyltrimethoxysilane. Test pieces were adhered and subjected to peel tensile tests to determine the best adhesion procedure. Adhesion tests were analyzed by using Fourier-transform infrared spectroscopy, scanning electron microscopy, and X-ray dispersive energy. The best result was achieved when surfaces were treated with trichloroisocyanuric acid. In conclusion, this treatment was the most effective in increasing adherence values in between PU sheets and in between sheets and elastomer layers.
\end{abstract}

Keywords: waste tire; oxidation; surface treatment; coupling agent 


\section{INTRODUCCIÓN}

El uso de recursos naturales, la globalización y el desarrollo de la sociedad moderna ha generado degradación de ecosistemas y complica la sostenibilidad (Gallego et al., 2020). La problemática referente a los residuos sólidos es uno de los aspectos que día a día se ven afectados con el aumento de la población, industrialización y la demanda de materias primas (Song et al., 2017). En particular, el problema de las llantas desechadas a nivel mundial se complica no solo por su volumen pues también influye su composición, debido a que es un material polimérico que no es degradado fácilmente, lo cual lleva a situaciones en las que se llegan a almacenar grandes cantidades de este residuo, y aunque existen variadas alternativas de tratamiento, ninguna es lo suficientemente atractiva para que los procesos de reaprovechamiento permitan emplear la cantidad disponible de llantas en desuso; tan solo en China en el año 2018 se generaron 14.60 millones de toneladas de llantas para auto usadas, cifra equivalente a 3.24 veces lo generado por la Unión Europea y 2.81 veces lo generado en Estados Unidos (Chen et al., 2021).

En la actualidad se pueden utilizar diversos métodos para la recuperación de neumáticos y la destrucción de sus componentes peligrosos. Los esfuerzos de la comunidad científica para encontrar formas de reducir los residuos de neumáticos han llevado a una intensa investigación sobre el caucho que incluye la posibilidad de aplicarlo en el relleno de concreto en vulcanizados de caucho natural y mezclas con polímeros (Colom et al., 2007; Manchón et al., 2004), también se ha utilizado caucho de llantas en desuso para intentar mejorar propiedades térmicas y mecánicas de biopolímeros como el ácido poliláctico con grafeno como microrelleno (Bijarimi et al., 2021), para incrementar la resistividad térmica, la absorción acústica y la amortiguación de vibraciones en compuestos de concreto (Bala y Gupta, 2021) y en la elaboración de materiales para el aislamiento térmico (Hittini et al., 2021). La superficie de láminas elaboradas a partir de llantas usadas se puede modificar a través de tratamientos como lo es el químico, la polimerización por injerto, modificación por radiación y modificación con gas (Romero et al., 2005; García et al., 2010). El objetivo de estos métodos es oxidar la superficie para formar grupos como el peroxi, hidroperoxi, hidroxilo y carbonilo. La presencia de estos grupos en la superficie mejora la interacción con los polímeros polares (Ramarad et al., 2015).

El proceso químico de oxidación se lleva a cabo utilizando componentes como el permanganato de potasio, ácido nítrico, ácido sulfúrico o peróxido de hidrógeno. Estos productos químicos actúan sobre la superficie de caucho de las Ilantas causando el grabado en la superficie y mejorando la adhesión mecánica entre el caucho y la matriz termoplástica (Li et al., 2016; Rungrodnimitchai y Kotatha, 2015). Sonnier et al (2007) utilizaron permanganato de potasio para romper los enlaces de caucho insaturados y crear grupos hidroxilo. Colom (2007) trataron la superficie de llanta con ácido sulfúrico y nítrico estableciendo que las propiedades de la mezcla mejoraron debido a una mayor humectación entre la superficie de llanta y la matriz polimérica. De igual forma, los silanos son reconocidos como agentes de acoplamiento eficientes ampliamente utilizados en materiales compuestos y formulaciones adhesivas (Bengtsson y Oksman, 2006). Se han aplicado con éxito en compuestos de polímeros reforzados con relleno inorgánico, como los compuestos de polímero reforzados con fibra de vidrio y compuestos de polímero con relleno de minerales (Xie et al., 2010). Los silanos también son promotores de la adhesión en muchas formulaciones adhesivas o se usan como primers de sustrato, lo que brinda una adhesión más fuerte (Deyá, 2016). Su estructura química está dada por la ecuación (1) siendo $R$ un grupo alcoxi, $X$ un grupo funcional y $R^{\prime}$ un puente de alquilo que conecta el átomo de silicio y el grupo funcional.

$$
R_{(4-n)}-S_{i}-\left(R^{\prime} X\right)_{n}(n=1,2)
$$

El mecanismo general para mejorar la adhesión se debe a la formación de un enlace covalente entre el silanol, como resultado de la hidrólisis del silano y la capa de hidroxilo presente en el sustrato. Por otro lado, el grupo funcional del silano se acopla con las matrices poliméricas de acuerdo con sus formas, esto dependiendo de la reactividad o compatibilidad de los grupos funcionales hacia el polímero (Sonnier et al., 2007; Rossi et al., 2017).

En el presente trabajo de investigación se realizaron varios procedimientos para mejorar la adherencia entre láminas elaboradas con llantas de desecho unidas con un adhesivo de poliuretano (PU) y la adherencia entre las láminas y un recubrimiento elastómerico (pintura) con el objetivo de evaluar los procedimientos de tratamiento superficial con ácido tricloroisocianúrico (ATI) o permanganato de potasio. Aunque ya existen estudios de tratamientos implementados en el caucho de llanta en desuso, el estudio de tratamiento con permanganato de potasio seguido del uso de silanos es una novedad. También se trató la superficie de la llanta con permanganato de potasio con el objeto de aumentar la cantidad de grupos hidroxilos, de esta manera, se esperaría que el silano 3-aminopropiltrietoxisilano (APT) o viniltrimetoxisilano (VTM) establezca enlaces covalentes fuertes entre la superficie de llanta y el PU.

\section{METODOLOGÍA}

Para la realización de las pruebas experimentales se utilizaron llantas en desuso de la marca Bridgestone referencia Dueler H/T 684 II Radial P225/60R7 98T. En cuanto a reactivos se empleó tolueno, ácido 
tricloroisocianúrico al 90\% en forma granular de color blanco, metil-etil-cetona (MEK), diisocianato de tolueno (TDI) comercializado por Pegaucho ${ }^{\circledR}$ (Bogotá-Colombia) como agente promotor de adhesión, silano APT y silano VTM (producidos por Struktol ${ }^{\circledR}$ Inc), permanganato de potasio grado analítico, ácido sulfúrico, peróxido de hidrógeno al $50 \%$, acetona, adhesivo de poliuretano en dispersion acuosa Hidropul $500^{\circledR}$ y recubrimiento elastómerico para exteriores de color blanco (pintura Paraguas ${ }^{\circledR}$ producida por Corona). Se llevaron a cabo tratamientos superficiales con ácido tricloroisocianúrico, diisocianato de tolueno, silanos (APT y VTM) y con permanganato de potasio. Con el caucho de llanta en desuso se elaboraron probetas rectangulares de $23 \mathrm{~mm}$ de ancho y $65 \mathrm{~mm}$ de largo cuyas superficies fueron sometidas a tratamiento abrasivo con esmeril con rueda de carburo de silicio grano 60 .

\section{Tratamiento con ATI y TDI}

Se disolvieron $2 \mathrm{~g}$ de ATI en $100 \mathrm{~g}$ de tolueno agitando vigorosamente durante 2 minutos. Después de dos horas de reposo de la solución se aplicaron dos capas (cada película seca con un peso de $0.013 \mathrm{~g}$ ) sobre la superficie de la probeta con un intervalo de cinco minutos entre cada capa y a una temperatura de $23^{\circ} \mathrm{C}$. Para el tratamiento con diisocianato un $1 \mathrm{~g}$ de TDI fue disuelto en $50 \mathrm{~g}$ de MEK, luego se aplicaron dos capas de esta solución sobre la superficie del caucho de llanta dejando un intervalo de cinco minutos entre cada aplicación.

\section{Tratamientos con APT y VTM}

Se mezclaron $200 \mathrm{~g}$ de etanol con $50 \mathrm{~g}$ de agua destilada. A esta solución se le agregaron $15 \mathrm{~g}$ de APT o VTM (según el caso) y se agitó durante 10 minutos. Durante $1 \mathrm{~h}$ (que es el tiempo de reacción de hidrolisis de los silanos) el pH se controló mediante la adición de ácido acético glacial y se mantuvo en $4.2 \pm 0.2$ y $3.2 \pm 0.2$ para APT y VTM respectivamente. A continuación, las probetas se sumergieron en la solución hidrolizada durante 4 horas a $23^{\circ} \mathrm{C}$, transcurrido este tiempo se lavaron con abundante agua destilada y se secaron por 24 horas a $55^{\circ} \mathrm{C}$ en un horno de convección forzada.

\section{Tratamientos con permanganato de potasio}

Para obtener la solución al 1\% p/v de $\mathrm{KMnO} 4$, en una solución de $270 \mathrm{~g}$ de agua destilada/23.4 g de acetona se disolvieron $3 \mathrm{~g}$ de permanganato de potasio. En esta solución se pusieron en inmersión las probetas durante cuatro horas a $23{ }^{\circ} \mathrm{C}$. Luego se lavaron las probetas, primero con una solución de peróxido de hidrogeno al $50 \%$, después con una solución de ácido sulfúrico al $0.1 \%$ y por último se enjuagaron con agua destilada. Para las soluciones al $2 \%$ y $3 \% \mathrm{p} / \mathrm{v}$ de $\mathrm{KMnO}_{4}$ se siguió el mismo procedimiento, pero incrementando la cantidad de permanganato a $6 \mathrm{~g}$ y $9 \mathrm{~g}$ respectivamente.

\section{Elaboración de juntas adhesivas}

Una vez sometidas las probetas al tratamiento superficial, con pares de ellas se elaboraron las juntas adhesivas. Para ello se les aplicó el adhesivo de PU y para secar la película se dejaron en un horno a $55^{\circ} \mathrm{C}$ durante 4 min (obteniendo películas de $0.09 \mathrm{~g}$ en base seca) dejándolas luego en reposo durante dos horas. Posteriormente las películas de adhesivo se reactivaron mediante calentamiento en un horno a $120^{\circ} \mathrm{C}$ durante dos minutos, se retiraron del horno y rápidamente (en un lapso no superior a 15 segundos) se unieron primero de forma manual e inmediatamente se presionaron mediante una prensa hidráulica con embolo de diámetro $14 \mathrm{~cm}$ a una presión de $5.516 \mathrm{MPa}$ (800 psi) durante 20 segundos. Para medir la fuerza adhesiva de las juntas se realizaron pruebas de adherencia ( $T$ peel) siguiendo los lineamientos establecidos en la norma ASTM C794-15a. Se utilizó una máquina universal de ensayos Shimadzu ${ }^{\circledR}$ AGS-X con velocidad de desplazamiento de $50 \mathrm{~mm} / \mathrm{min}$, temperatura de $24^{\circ} \mathrm{C}$ y humedad relativa de $54 \%$.

\section{Recubrimiento de las probetas tratadas}

En cuanto al pintado de las probetas, mediante un aerógrafo se aplicaron dos capas de recubrimiento elastómerico a $20 \mathrm{~cm}$ de distancia. Entre cada aplicación transcurrió una hora para el secado. Para la medición de la fuerza de adhesión de la capa de pintura sobre la superficie de la probeta siguió el procedimiento descrito en la Norma ASTM D3357-17.

Para la realización de las pruebas experimentales se recurrió a un diseño de un factor con 11 tratamientos (uno de los cuales corresponde al blanco, es decir sin modificación superficial), cada uno de ellos con tres replicas, para así poder realizar el correspondiente análisis de varianza (ANOVA) y posteriormente la prueba de Tukey de comparación entre las medias de los tratamientos. El procesamiento de la información y la obtención de los estadísticos para el análisis se hizo mediante Excel ${ }^{\circledR}$ estableciendo un valor $\alpha=0.05$. En cuanto a la nomenclatura de los tratamientos, el termino ST significa sin tratamiento, TDI es tratamiento con diisocianato de tolueno, APT con 3-aminopropiltrietoxisilano, VTM con viniltrimetoxisilano y ATI con ácido tricloroisocianúrico. $\mathrm{K} 1$, $\mathrm{K} 2$ y K3 simbolizan tratamientos con permanganato de potasio al $1 \%, 2 \%$ y $3 \%$ en p/v respectivamente, mientras que las siglas $\mathrm{K} 1 \mathrm{~A}, \mathrm{~K} 2 \mathrm{~A}$ y $\mathrm{K} 3 \mathrm{~A}$ se refieren a los tres tratamientos efectuados con 
permanganato de potasio (al 1\%, $2 \%$ y $3 \%$ ) y 3-aminopropiltrietoxisilano. Finalmente se caracterizaron las superficies por espectroscopia FT-IR en un espectrómetro Thermo Scientific Nicolet ${ }^{\circledR}$ iS10 FT-IR con una resolución mejor que $0.4 \mathrm{~cm}^{-1}$, el método de análisis fue mediante el uso del módulo de Reflexión Total Atenuada (ATR). La morfología de las superficies fue analizada mediante un microscopio electrónico de barrido JEOL ${ }^{\circledR}$ modelo JSM 6490-LV.

\section{RESULTADOS}

Ensayos de adherencia en juntas de caucho de llanta reciclado con poliuretano como adhesivo se efectuaron para determinar las propiedades de adhesión. En la Figura 1 se muestran los valores obtenidos en las pruebas de adherencia de los diferentes métodos llevados a cabo en este estudio. Al realizar el correspondiente análisis de varianza, el valor $p$ obtenido $\left(1.88{ }^{*} 10^{-13}\right)$ indica que hay diferencia significativa entre los tratamientos. La posterior aplicación de la prueba de Tukey de comparación entre medias con $\alpha=0.05$ permite dividir claramente los tratamientos en tres grupos (que se pueden apreciar en la Figura 1); en el primero se encuentran ST, TDI, APT, VTM y K2, el segundo están K1, K1A, K2A, K3, K3A y el tercero está constituido solamente por el tratamiento ATI. No hay diferencia significativa entre tratamientos de un mismo grupo, pero si la hay entre tratamientos de grupos diferentes.

Los valores correspondientes a tratamiento con TDI, tratamiento con APT, con VTM y K2 se pueden considerar de baja adherencia, de hecho, no hay diferencia significativa en sus promedios de adherencia respecto a no tratar la superficie de la probeta (ST). Por otro lado, el valor más alto, con una media de 3.52 $\mathrm{KN} / \mathrm{m}$, se consiguió con el método de modificación de superficie con ATI, que está acorde con valores conseguidos por estudios realizados en otras publicaciones (Song et al., 2017; García et al., 2010; Radabutra et al., 2009).

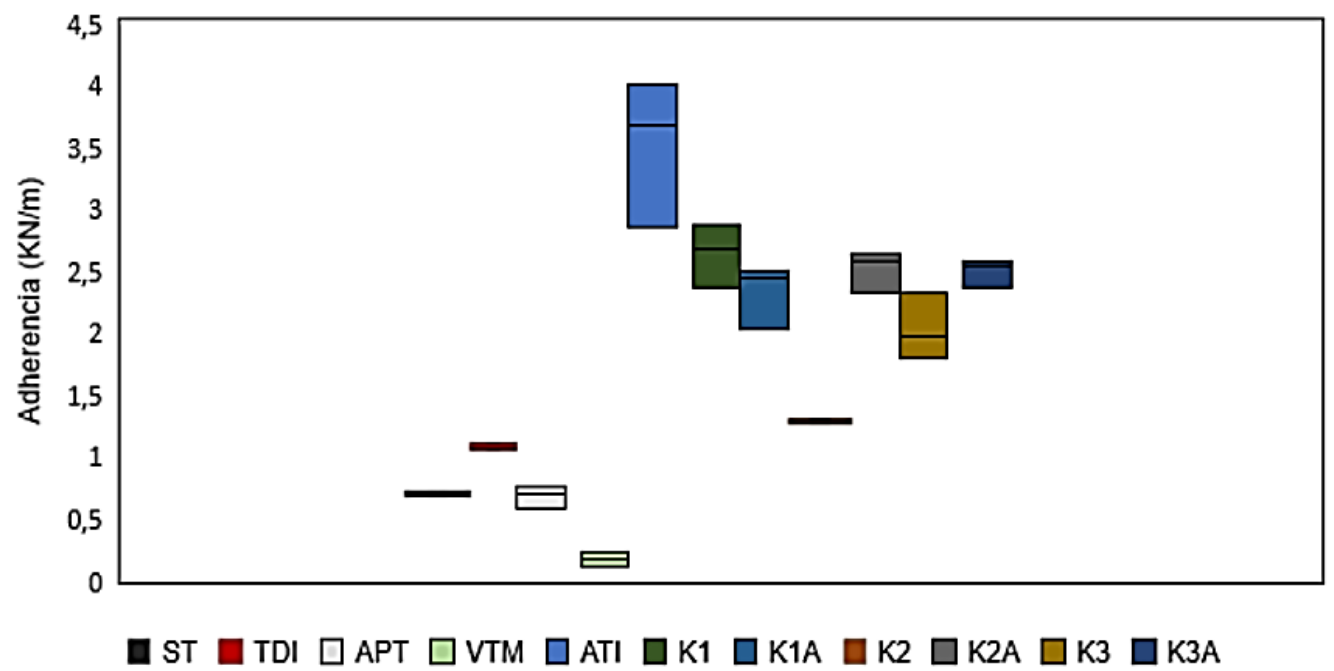

Fig. 1: Adherencia obtenida con los diferentes tratamientos analizados

En la franja de valores que corresponde a tratamientos de oxidación con permanganato de potasio, es llamativo que no hay diferencias significativas entre ellos, salvo con K2. Se aprecia que el valor más alto alcanzado, con una media de $2.64 \mathrm{KN} / \mathrm{m}$, es el que corresponde al tratamiento con permanganato de potasio al $1 \%$ (K1), que a su vez es $24.9 \%$ más bajo que el tratamiento ATI. El reforzamiento de la adhesión con el uso de silano APT, no provoco un incremento en el valor de la adhesión con respecto al caso en que se usó $\mathrm{K} 1$, sin embargo, los métodos en que se aplicó tratamiento con permanganato de potasio al $2 \%$ con silano APT (K2A) y tratamiento con permanganato de potasio al 3\% con silano APT (K3A) lograron incrementar este valor con respecto a $\mathrm{K} 2$ en $48.7 \%$ y $51.26 \%$ respectivamente. En estos casos el silano logró incrementar el valor de la fuerza de adhesión, pero en general se aprecia que la fuerza de adhesión con o sin silano APT no supera el valor que se obtuvo con K1 (Stapleton et al., 2012).

Por otra parte, en la Tabla 1 se muestran los resultados correspondientes a las pruebas realizadas para el análisis de adhesión de la pintura sobre las probetas. Se aprecian los diferentes valores de la clasificación de la prueba de adhesión del elastómero, desde 5B hasta $0 \mathrm{~B}$, que representa un porcentaje de remoción de elastómero de la superficie que va desde $0 \%$ hasta un valor de $65 \%$ o mayor. Se observa que las probetas relacionadas como ST, VTMS y APT presentan un resultado equivalente a una valoración de 0B. El resto de las pruebas relacionadas en la tabla 1: ATI, K1, K2, K3, K1A, K2A y K3A, tienen un resultado de 5B. Se puede observar que no hay valores intermedios, y que, teniendo en cuenta los resultados de los dos tipos de pruebas, el tratamiento superficial con ATI es exitoso tanto para la adherencia entre probetas elaboradas con llantas en desuso como para adherencia de pintura a las probetas. 
Tabla 1: Resultados de los ensayos de análisis de adherencia del recubrimiento elastomérico.

\begin{tabular}{|c|c|c|c|c|c|c|}
\hline Clasificación & $5 B$ & $4 B$ & $3 B$ & $2 B$ & $1 B$ & $0 B$ \\
\hline $\begin{array}{c}\text { Porcentaje de } \\
\text { área removida }\end{array}$ & $0 \%$ & $<5 \%$ & Entre $5 \%$ y $15 \%$ & Entre 15 y $35 \%$ & Entre 35 y $65 \%$ & $>65 \%$ \\
\hline $\begin{array}{c}\text { Resultado de } \\
\text { las pruebas }\end{array}$ & $\begin{array}{c}\mathrm{ATI}, \mathrm{K} 1, \mathrm{~K} 2, \mathrm{~K} 3, \\
\mathrm{~K} 1 \mathrm{~A}, \mathrm{~K} 2 \mathrm{~A}, \mathrm{~K} 3 \mathrm{~A} .\end{array}$ & & & & & $\begin{array}{c}\text { ST, VTM, } \\
\text { APT. }\end{array}$ \\
\hline
\end{tabular}

Para el análisis por FT-IR, en la Figura 2 se muestran los resultados de los espectros de los tratamientos superficiales que se realizaron en este estudio. El espectro que corresponde a la superficie del caucho de Ilanta reciclada sin tratamiento (Figura 2, ST), presenta una señal en $2915 \mathrm{~cm}^{-1}$ que pertenece al enlace alifático C-H debido al estiramiento asimétrico por vibración del grupo $-\mathrm{CH}_{3}$. También en $2845 \mathrm{~cm}^{-1}$ se aprecia una señal del enlace alifático $\mathrm{C}-\mathrm{H}$ provocado por un estiramiento simétrico por vibración de los grupos $-\mathrm{CH}_{2}$. La banda de $1535 \mathrm{~cm}^{-1}$ indica un pico característico de un estiramiento de un doble enlace $\mathrm{C}=\mathrm{C}$, proveniente del negro de humo, por último, la señal en $1434 \mathrm{~cm}^{-1}$ se debe a una vibración tipo tijera del $-\mathrm{CH}_{2}-$ afectado por los átomos de $\mathrm{S}$ en el grupo $-\mathrm{CH}_{2}-\mathrm{S}-\mathrm{CH}_{2}$ (Ramarad et al., 2015).

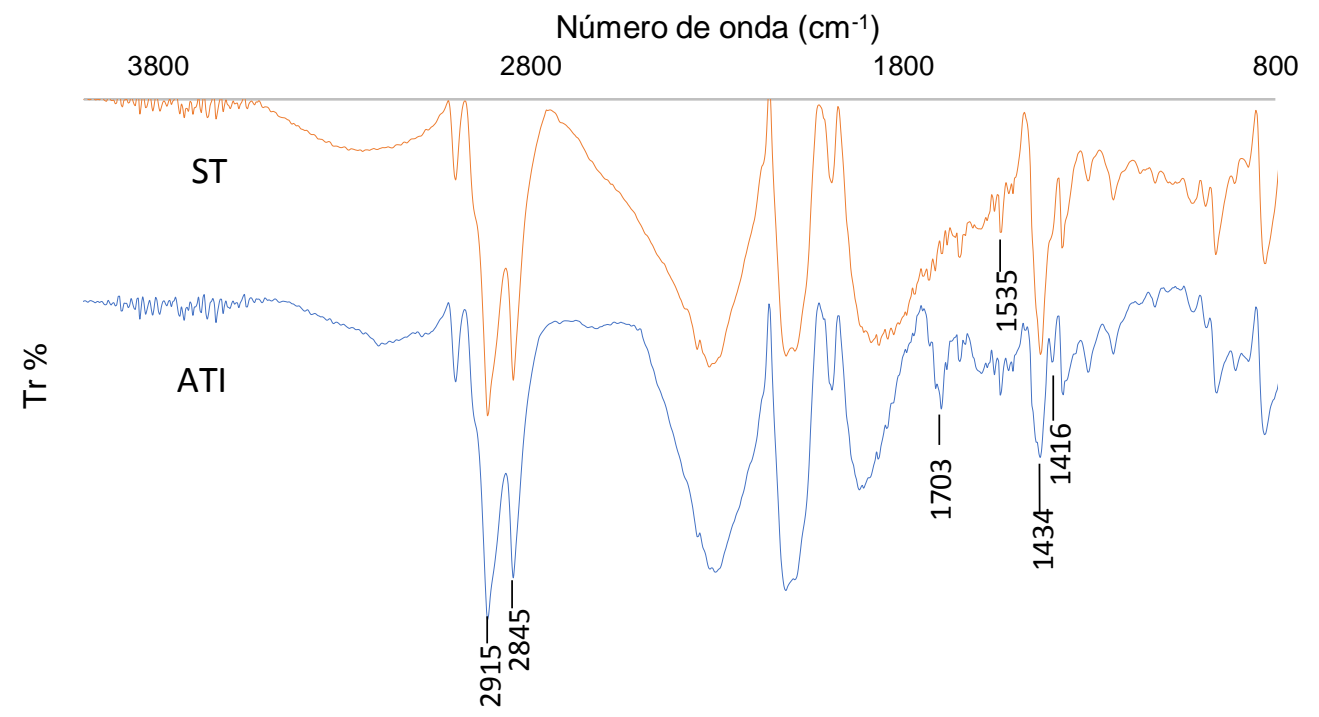

Fig. 2: FT-IR correspondiente a las pruebas ST y ATI.

La Figura 2 (ATI) ilustra el tratamiento de la superficie de llanta con ATI, se aprecia la banda de $1416 \mathrm{~cm}^{-1}$ perteneciente a la deformación por flexión del halógeno, también la banda en $1703 \mathrm{~cm}^{-1}$ atribuido a la

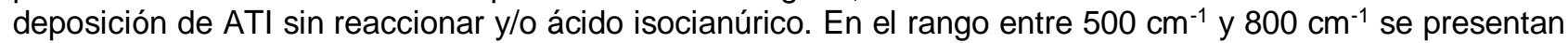
las señales características para los enlaces $\mathrm{C}-\mathrm{Cl}$ pero estas se encuentran dentro de la señal de ruido del espectro IR por lo tanto no se pueden analizar (Romero et al., 2005; García et al., 2010; Tyczkowski et al., 2009).

En cuanto a los tratamientos con permanganato de potasio, el espectro mostrado en la Figura 3 (K2) indica como la intensidad de los picos ubicados en 2840-2940 y $1630 \mathrm{~cm}^{-1}$, que están relacionados con las vibraciones de estiramiento de los grupos $-\mathrm{CH}_{2}$ y $-\mathrm{C}=\mathrm{O}$ respectivamente, aumenta, lo que permitiría suponer un aumento de estos grupos sobre la superficie de la muestra. Sin embargo, la intensidad relativa de las vibraciones de estiramiento de $-\mathrm{OH}$ a aproximadamente $3340 \mathrm{~cm}^{-1}$ se mantiene equivalente, esto debido probablemente a que la superficie absorbe humedad del ambiente e interfiere con su valor real (Delor et al., 1998). Los espectros FT-IR de la Figura 3 pertenecen a los resultados obtenidos después de los tratamientos K2 y K2A. En ellos se aprecia el pico a $1092 \mathrm{~cm}^{-1}$, el cual se relaciona con grupos de $\mathrm{Si}-\mathrm{O}-\mathrm{CH}_{3}$ residuales no hidrolizables. El pico alrededor de $1192 \mathrm{~cm}^{-1}$ se debe al estiramiento de los enlaces Si-O-Si y Si-O-C (Abdelmouleh et al., 2007). Es decir, el pico a $1192 \mathrm{~cm}^{-1}$ indica la posible unión covalente entre el silano y los grupos - $\mathrm{OH}$ que se formaron sobre la superficie de la llanta después de la oxidación con permanganato de potasio. En el análisis complementario por microscopia electrónica de barrido (SEM), las micrografías correspondientes a las superficies de las muestras con el tratamiento K2 y K2A se ilustran en las Figuras 4 y 5 respectivamente.

En la Figura 4, se puede ver como la humectación de PU es más acentuada en la superficie de la probeta con tratamiento K2A que en la superficie de la probeta K2, esto debido posiblemente a que hay un aporte extra en la fuerza de adhesión por parte del silano. La Figura 5 permite reforzar el hecho de que la humectación de PU es más notoria en la superficie de las láminas elaboradas con caucho de llanta usada si se aplica el tratamiento K2A que si se recurre al tratamiento K2. 


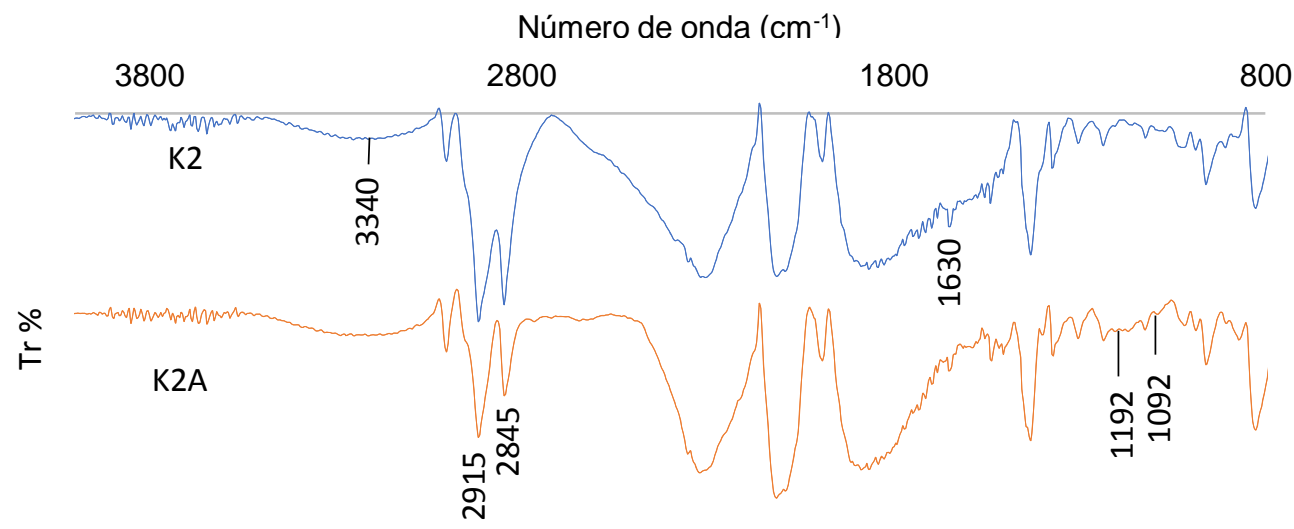

Fig. 3: FT-IR correspondiente a las pruebas K2 y K2A.

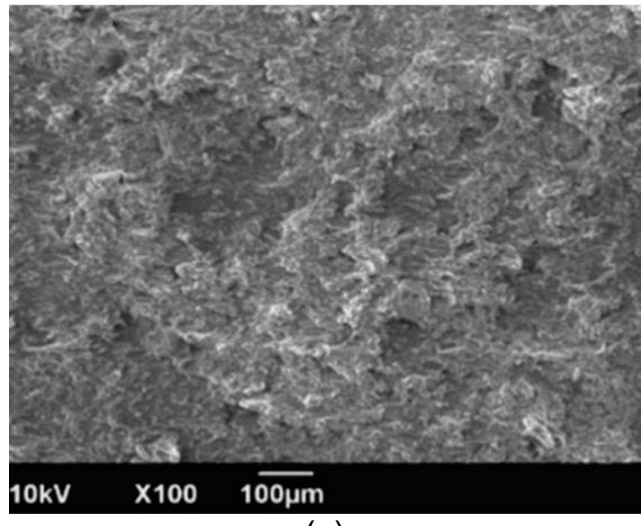

(a)

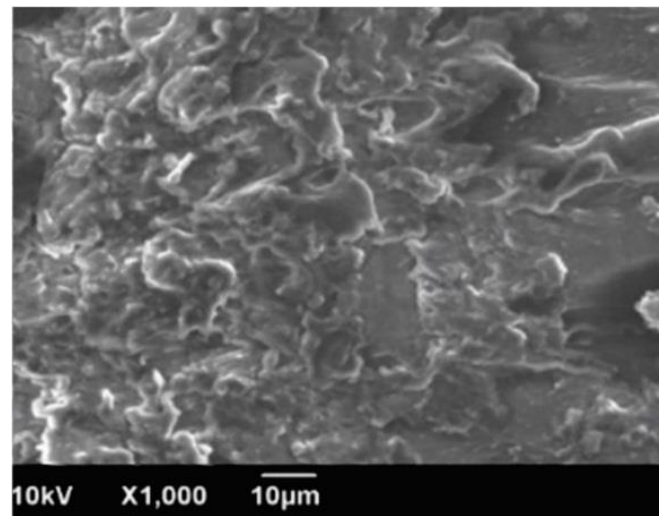

(b)

Fig. 4: SEM correspondiente a la superficie de K2. (a) equivalente a x100 y (b) equivalente a x1000 del tamaño real.

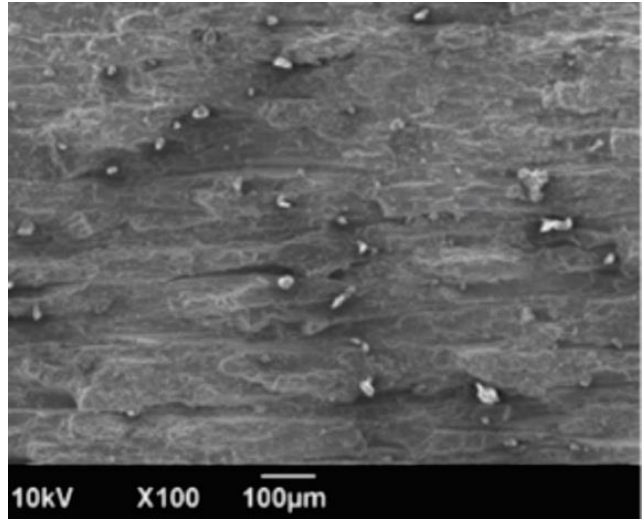

(a)

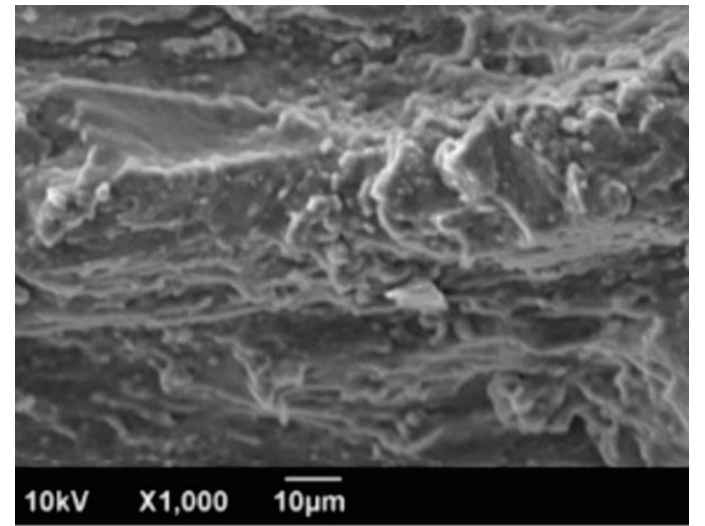

(b)

Fig. 5: SEM correspondiente a la superficie de K2A. (a) equivalente a x100 y (b) equivalente a x1000 del tamaño real.

Por otra parte, la Figura 6 permite ver la interfase formada entre la superficie de las láminas elaboradas con caucho proveniente de llantas para automóvil usadas y el elastómero empleado. En ella, se aprecia el estiramiento del elastómero (indicado por la flecha), lo que supone una fuerte adherencia a la superficie de las probetas elaboradas con el caucho proveniente de llanta usada (Fang et al., 2014). Los valores que se presentan en la Tabla 2 se refieren a los porcentajes en peso de los elementos detectados en la superficie de la llanta mediante la aplicación del método de energía dispersiva de rayos X (EDX). La superficie de llanta lisa presenta los valores propios de una llanta usada común de auto.

La superficie de la llanta sin tratamiento (ST) más PU proporciona datos con un aumento en el valor de porcentaje de peso de oxígeno (respecto a la probeta ST) debido al aporte de poliuretano, lo mismo sucede con el tratamiento K2 en el cual el porcentaje de oxígeno es mayor, posiblemente debido al aporte que proporciona el proceso de oxidación con permanganato de potasio. El resultado del tratamiento K2A presenta un valor más alto en composición de silicio que el obtenido en K2, seguramente debido al aporte del silano utilizado. 


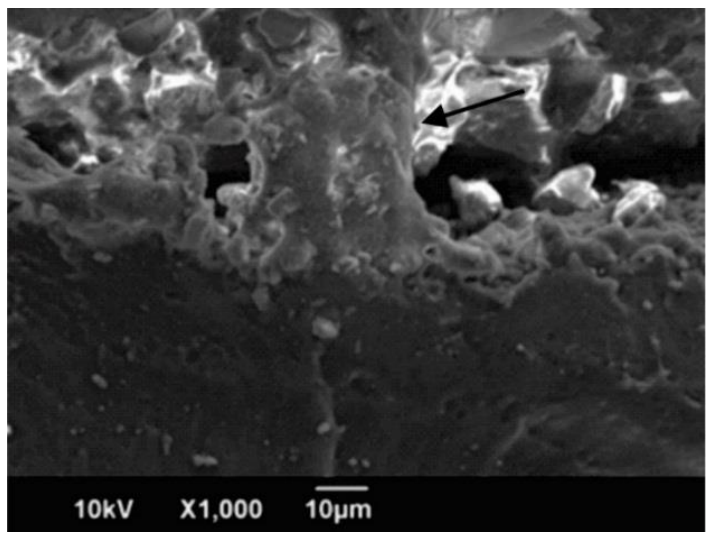

Fig. 6: SEM correspondiente a la superficie cubierta con elastómero.

Tabla 2: EDX superficie de las probetas. Análisis elemental (porcentajes en peso).

\begin{tabular}{|c|c|c|c|c|}
\hline & $S T$ & $S T+P U$ & $K 2$ & $K 2 A$ \\
\hline $\mathrm{C}$ & 92.52 & 88.71 & 70.91 & 90.02 \\
\hline $\mathrm{O}$ & 4.07 & 8.20 & 27.88 & 6.94 \\
\hline $\mathrm{S}$ & 2.50 & 1.46 & 0.52 & 2.30 \\
\hline $\mathrm{Cl}$ & 0.0 & 0.56 & 0.0 & 0.0 \\
\hline $\mathrm{Zn}$ & 0.92 & 1.07 & 0.0 & 0.0 \\
\hline $\mathrm{Si}$ & 0.0 & 0.0 & 0.26 & 0.42 \\
\hline $\mathrm{Na}$ & 0.0 & 0.0 & 0.44 & 0.32 \\
\hline
\end{tabular}

\section{DISCUSIÓN FINAL}

Se aplicaron diez tratamientos superficiales tendientes a mejorar la adhesión entre láminas elaboradas a partir de caucho proveniente de llantas para automóvil usadas y desechadas, a manera de contraste también se realizaron mediciones sobre probetas elaboradas con el mismo caucho, pero sin realizarles tratamiento alguno. Los resultados obtenidos después de la aplicación de cada método de adhesión y de la medición de las propiedades correspondientes, indican que el mejor resultado se obtuvo utilizando ácido tricloroisocianúrico (ATI) como modificador de la superficie, seguido de los tratamientos con permanganato de potasio (a excepción de K2). De ello se desprende que los valores de respuesta correspondientes al tratamiento con permanganato de potasio al $2 \%$ (denominado K2) son más bajos que los obtenidos con los restantes tratamientos con permanganato de potasio. Los primers TDI, APT y VTM no consiguieron un incremento significativo en la adhesión entre las láminas. El Silano APT logra crear enlaces covalentes Si-OSi y Si-O-C entre el PU y los grupos hidroxilo creados por la oxidación con permanganato de potasio en la superficie de las probetas elaboradas con caucho de llanta usada. Estos enlaces se corroboraron mediante análisis con FT-IR. El uso del recubrimiento elastómerico permite establecer que con un adhesivo apropiado se logran mejores resultados en los valores de adherencia.

\section{CONCLUSIONES}

De acuerdo con el trabajo presentado y a los resultados obtenidos, se pueden plantear las siguientes conclusiones principales:

1.- El tratamiento superficial con ácido tricloroisocianúrico (ATI) realizado a láminas elaboradas con caucho proveniente de llantas en desuso para automóviles, resulta efectivo para incrementar los valores de adherencia tanto entre láminas unidas mediante PU como entre láminas y recubrimiento elastómerico.

2.- Los tratamientos superficiales realizados mediante la utilización exclusiva de silanos (APT y VTM) no son efectivos para mejorar la adherencia entre láminas pegadas con PU ni para incrementar la adherencia entre las láminas y pintura elastómerica.

3.- Los tratamientos superficiales efectuados con permanganato de potasio ( $K 1, K 2, K 3, K 1 A, K 2 A, K 3 A)$ son apropiados para mejorar la adherencia entre las láminas de caucho y recubrimiento elastómerico. En cuanto a la unión entre láminas mediante PU, aunque permiten obtener una mejor adherencia (excepto K2) en comparación a no hacer tratamiento o a modificaciones superficiales con silanos, el análisis estadístico muestra que su desempeño es inferior al obtenido con el tratamiento ATI.

\section{AGRADECIMIENTOS}

Los autores agradecen a la Vicerrectoría de Investigaciones de la Universidad Militar Nueva Granada por el apoyo financiero a través del proyecto INV-ING-2627 vigencia 2018. 


\section{REFERENCIAS}

Abdelmouleh, M., Boufi, S., Belgacem, M., y Dufresne, A., Short natural-fibre reinforced polyethylene and natural rubber composites: Effect of silane coupling agents and fibres loading, Composites Science and Technology, 67, 1627-1639 (2007)

Bala, A., y Gupta, S., Thermal resistivity, sound absorption and vibration damping of concrete composite doped with waste tire Rubber: A review, https://doi.org/10.1016/j.conbuildmat.2021.123939, Construction and Building Materials, 299, 123939 (2021)

Bengtsson, M., y Oksman, K., The use of silane technology in crosslinking polyethylene/wood flour composites, https://doi.org/10.1016/j.compositesa.2005.06.014, Composites Part A: Applied Science and Manufacturing, 37, 752-765 (2006)

Bijarimi, M., Francisco, V., y otros 4 autores, Mechanical properties of poly(lactic acid) compounded with recycled tyre waste/graphene nanoplatelets nanocomposite, https://doi.org/10.1016/j.matpr.2021.01.498, Materials Today: Proceedings, 42, 265-269 (2021)

Chen, B., Zheng, D., y otros 9 autores, Disposal methods for used passenger car tires: One of the fastest growing solid wastes in China, https://doi.org/10.1016/j.gee.2021.02.003, Green Energy \& Environment, en prensa (2021)

Colom, X., Carrillo, F., y Cañavate, J., Composites reinforced with reused tyres: Surface oxidant treatment to improve the interfacial compatibility, https://doi.org/10.1016/j.compositesa.2006.01.022, Composites Part A: Applied Science and Manufacturing, 38, 44-50 (2007)

Delor, F., Barrois, N., y otros 4 autores., Oxidation of rubbers analysed by HATR/IR spectroscopy, Polymer Degradation and Stability, 62, 395-401 (1998)

Deyá, C., Silane as adhesion promoter in damaged areas, Progress in Organic Coatings, 90, 28-33 (2016)

Fang, L., Chang, L., y otros 3 autores, Influence of silane surface modification of veneer on interfacial adhesion of woodplastic plywood, Applied Surface Science, 288, 682- 689 (2014)

Gallego, L., Hernández, L., Orjuela, H., y Araque, O., Aplicación de la técnica de componentes principales en la determinación de variables de activos ambientales, http://dx.doi.org/10.4067/S0718-07642020000600125, Información Tecnológica, 31 (6), 125-132 (2020)

García, C., Andreu, V., y Martín, J., Surface modification of vulcanized styrene-butadiene rubber with trichloroisocyanuric acid solutions of different active chlorine contents, https://doi.org/10.1016/j.ijadhadh.2010.06.004, International Journal of Adhesion and Adhesives, 30, 550-558 (2010)

Hittini, W., Mourad, A., y Abu-Jdayil, B., Utilization of devulcanized waste rubber tire in development of heat insulation composite, https://doi.org/10.1016/j.jclepro.2020.124492, Journal of Cleaner Production, 280, 124492 (2021)

Li, W., Meng, L., y Ma, R., Effect of surface treatment with potassium permanganate on ultra-high molecular weight polyethylene fiber reinforced natural rubber composites, Polymer Testing, 55, 10-16 (2016)

Manchón, E., Macías, A., y otros 3 autores, Preparation of mesoporous and macroporous materials from rubber of tyre wastes, Microporous and Mesoporous Materials, 67, 35-41 (2004)

Radabutra, S., Thanawan, S., y Amornsakchai, T., Chlorination and characterization of natural rubber and its adhesion to nitrile rubber, European Polymer Journal, 45, 2017-2022 (2009)

Ramarad, S., Khalid, M., y otros 3 autores, Waste tire rubber in polymer blends: A review on the evolution, properties and future, Progress in Materials Science, 72, 100-140 (2015)

Romero, M., Pastor, M., y Martín, J., Environmental friendly surface treatments of styrene-butadiene-styrene rubber: alternatives to the solvent-based halogenation treatment, International Journal of Adhesion and Adhesives, 25, 19-29 (2005)

Rossi, C., Caputo, P., y otros 3 autores, Quantitative evaluation of organosilane-based adhesion promoter effect on bitumen-aggregate bond by contact angle test, International Journal of Adhesion and Adhesives, 72, 117-122 (2017)

Rungrodnimitchai, S., y Kotatha, D., Chemically modified ground tire rubber as fluoride ions adsorbents, Chemical Engineering Journal, 282, 161-169 (2015)

Song, P., Li, S., y Wang, S., Interfacial interaction between degraded ground tire rubber and polyethylene, Polymer Degradation and Stability, 143, 85-94 (2017)

Sonnier, R., Leroy, E., y otros 3 autores, Polyethylene/ground tyre rubber blends: Influence of particle morphology and oxidation on mechanical properties, Polymer Testing, ISSN 01429418, 26, 74-281 (2007)

Stapleton, S., Waas, A., y Arnold, S., Functionally graded adhesives for composite joints, https://doi.org/10.1016/j.ijadhadh.2011.11.010, International Journal of Adhesion and Adhesives, 35, 36-49 (2012)

Tyczkowski, J., Krawczyk, I., Wożniak, B., y Martin, J., Low-pressure plasma chlorination of styrene-butadiene block copolymer for improved adhesion to polyurethane adhesives, European Polymer Journal, 45, 1826-1835 (2009)

Xie, Y., Hill, C., y otros 3 autores, Silane coupling agents used for natural fiber/polymer composites: A review, https://doi.org/10.1016/j.compositesa.2010.03.005, Composites Part A: Applied Science and Manufacturing, 41, 806-819 (2010) 